\title{
Indoor Optical Wireless Communications using Few- mode Based Uniform Beam Shaping and LMS Based Adaptive Equalization
}

\author{
Jianghao Li \\ Department of Electrical and Electronic \\ Engineering \\ The University of Melbourne \\ Melbourne, VIC 3010, Australia \\ jianghaol1@student.unimelb.edu.au
}

\author{
Christina Lim \\ Department of Electrical and Electronic \\ Engineering \\ The University of Melbourne \\ Melbourne, VIC 3010, Australia \\ chrislim@unimelb.edu.au
}

\author{
Ampalavanapillai Nirmalathas \\ Department of Electrical and Electronic \\ Engineering \\ The University of Melbourne \\ Melbourne, VIC 3010, Australia \\ nirmalat@unimelb.edu.au
}

\begin{abstract}
An indoor optical wireless communication system employing few-mode based uniform beam shaping and adaptive LMS equalization is demonstrated. Results show that superior uniformity is obtained and LMS equalization with step size of 0.01 and tap length of 45 achieves the best performance.
\end{abstract}

\section{Keywords—optical wireless communication, few-mode, uniform beam shaping, least mean square, channel equalization}

\section{INTRODUCTION}

Optical wireless communications (OWC) is a promising solution to meet the unprecedented increasing demand for broadband applications [1]. However, there are many limitations in the realization of OWC. First, compared to radio frequency (RF) based communications, OWC is more vulnerable to link outage [2] and it is more difficult to develop multiuser communications because of the Gaussian-shaped beam laser which is widely used in OWC that results in narrow beam with non-uniform power distribution. Secondly, inter-symbol interference (ISI) is a significant problem that highly limit the transmission data rate in high-speed OWC $[3,4]$. To solve the issues mentioned above, we propose an indoor optical wireless communication system using few-mode based uniform beam shaping and least mean square (LMS) based adaptive equalization. A data rate of $10 \mathrm{~Gb} / \mathrm{s}$ transmission were achieved and successfully received over the uniform beam. The experimental results show that superior uniformity with variation $\leqslant 4.1 \%$ across the beam can be generated and the best bit-error rate (BER) performance can be achieved by using LMS equalization with step size of 0.01 and tap length of 45 .

\section{EXPERIMENTAL SETUP}

The experimental setup is shown in Fig.1. At the transmitter, $10 \mathrm{~Gb} / \mathrm{s}$ on-off keying (OOK) data was generated using an arbitrary waveform generator (AWG). After amplified using by two electrical amplifiers (EAs), the data streams were used to drive two MachZehnder modulators (MZMs) to generate the OOK optical signals. Two external cavity lasers (LDs) at wavelengths of $1549 \mathrm{~nm}$ and $1551 \mathrm{~nm}$ respectively, were used as the optical sources. $\mathrm{LP}_{11 \mathrm{a}}$ and $\mathrm{LP}_{11 \mathrm{~b}}$ modes were generated using a photonic lantern (PL) mode multiplexer after the signals were amplified by an erbium-doped fiber amplifier (EDFA). The donut-shaped beam was attained by adjusting the polarization controls (PC3-6), where PC5 and PC6 were used with few-mode fibers (FMFs) to control the polarization of the $\mathrm{LP}_{11}$ modes. After that, the donut beam and the $\mathrm{LP}_{01}$ beam were superposed using a 50:50 beam splitter to obtain a uniform beam. The transmitted few-mode optical signals were coupled into FMF using a custom-designed lens system and then demultiplexed into 3 branches of $\mathrm{LP}_{01}$ mode signals by a PL at the receiver. After being amplified by 3 EDFAs, the optical signals were received using respective photodetectors. Finally, the recovered electrical signals were sampled by the real-time digital signal oscilloscope (DSO) for offline digital signal processing.

The detailed principle of LMS based equalization has been described in [5]. In the experiment, the 3 received signals from the 3 modes are fed into 3 adaptive finite-impulse-response (FIR) filters. Each FIR filter has $L$ tap numbers described by the coefficient

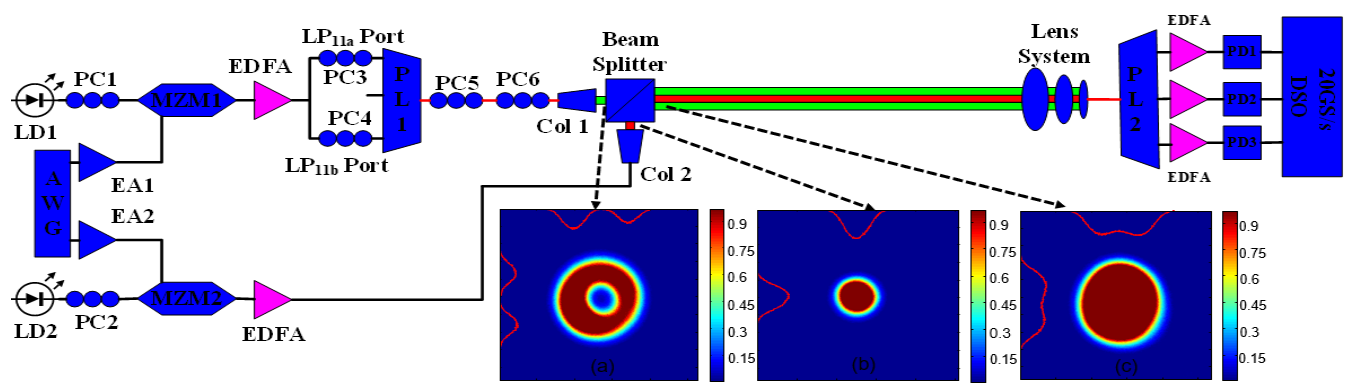

Fig. 1. Experimental setup and CCD images of the intensity distribution of (a) donut-shaped $\mathrm{LP}_{11}$ mode; (b) $\mathrm{LP}_{01}$ mode and (c) combined uniform beam.

This work was supported by Australian Research Council Discovery Project DP170100268. 


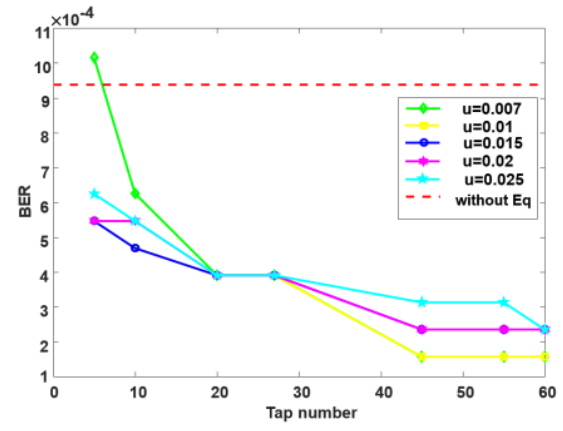

(a)
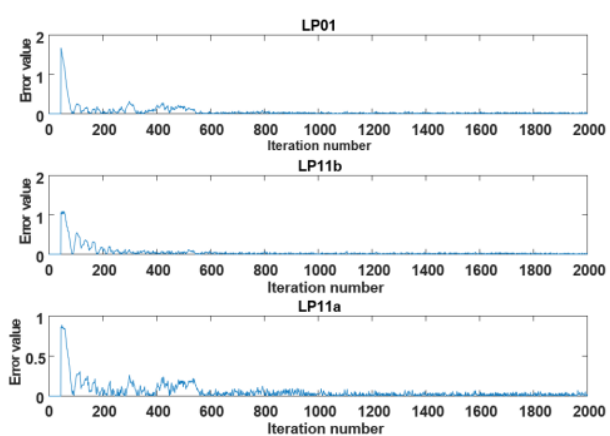

(b)
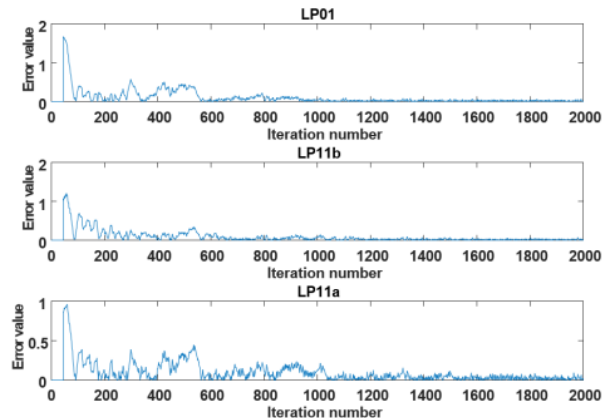

(c)

Fig. 2. (a) BER performance; (b) The error values vs. different iteration number $(\mu=0.01)$; (c) The error values vs. different iteration number ( $\mu=0.007$ ).

vectors $\mathbf{w}_{i}(i=1,2,3)$ with length $L$ and each FIR filter produces an output signal $y_{\mathrm{i}}(k)$ expressed as

$$
y_{i}(k)=\sum_{n=1}^{L} \mathbf{w}_{i}^{T}(k) \mathbf{x}_{i}(k)
$$

where $\mathbf{x}_{i}(k)$ is the $k$ th symbol of the $i$ th received signal and $\mathbf{w}_{i}(k)$ represents the $k$ th symbol of the $i$ th symbol of $\mathbf{w}_{i} . \mathbf{w}_{i}$ is updated continuously by employing the LMS algorithm, expressed as

$$
\mathbf{w}_{i}(k+1)=\mathbf{w}_{i}(k)+2 \mu\left[d_{i}(k)-y_{i}(k)\right] \mathbf{x}_{i}(k),
$$

where $\mu$ is the step size and $d_{i}(k)$ is the $k$ th desired output of the $i$ th equalizer. The coefficients were first updated using data-aided algorithm and then switched to decision directed algorithm after convergence. The equalized signals were then combined using maximum ratio combining (MRC) algorithm with weight coefficients $\alpha_{i}=P_{\mathrm{i}} / P$, where $P_{i}$ is the power of the $i$ th branch of the received signals and $P$ is the total power of the 3 signals. Finally, the combined signals were converted into binary symbols for BER calculation subject to hard decision.

\section{EXPERIMENTAL RESULTS AND DISCUSSIONS}

The beam profiles of the outputs were captured using a charge coupled device (CCD) camera at collimator 1 (Col.1), Col.2 and the beam splitter as shown in Fig.1 (a)-(c), respectively. The measured profiles showed high-quality uniform beam (variation $\leqslant$ $4.1 \%$ across the beam) can be obtained after superposing the $\mathrm{LP}_{01}$ and the donut-shaped $\mathrm{LP}_{11}$ beam. The BER performance of the OWC system with and without LMS equalization is shown as Fig.2 (a). The BER performance can be improved by increasing the tap number. When tap numbers are larger than 10, all the BER performance with different step length is better than that without LMS equalization. The best BER performance can be achieved when step lengths are 0.007 and 0.01 and tap number is larger than 45. The error values of the OWC system from different modes when $\mu=0.01$ and 0.007 are shown as Fig 2. (b) and (c), respectively. It can be found that the convergence speed of the signals from $L P_{11 a}$ channel is much slower than those of $\mathrm{LP}_{01}$ and $\mathrm{LP}_{11 \mathrm{~b}}$ channels. Moreover, the required iteration number of $\mathrm{LP}_{11 \mathrm{a}}$ channel until the desired convergence is about 600 when $\mu=0.01$ while the iteration number of the channel is more than 1000 when $\mu=0.007$. Considering the BER performance, computational complexity and convergence speed, the LMS based equalization with step size of 0.01 and tap length of 45 achieves the best performance.

\section{CONCLUSIONS}

In this paper, few-mode uniform beam shaping and LMS based adaptive equalization have been proposed and experimentally demonstrated for indoor OWC to alleviate the system performance degradation caused by link outage and ISI, respectively. The output beam can achieve a uniform power level with variation less than $4.1 \%$. The best performance can be achieved using LMS based adaptive equalization when step size is 0.01 and tap number is 45 .

\section{REFERENCES}

[1] A. Nirmalathas et al., "High-speed optical wireless communications for local area networks ," in Proc. Opto-Electron. Commun. Conf., 2018, pp. 1-2.

[2] T. Koonen, "Indoor optical wireless systems: Technology, trends, and applications," J. Lightw. Technol., vol. 36, no. 8, pp. 1459-1647, Apr. 2018.

[3] G. Cossu, A. M. Khalid, P. Choudhury, R. Corsini, and E. Ciaramella, "3.4 Gbit/s visible optical wireless transmission based on RGB LED," Opt. Express 20(26), B501-B506 (2012).

[4] C. Gong and Z. Xu, "Channel Estimation and Signal Detection for Optical Wireless Scattering Communication With Inter-Symbol Interference," IEEE Trans. Wireless Commun., vol. 14, no. 10, pp. 5326-5337, Oct. 2015.

[5] N. Benvenuto and G. Cherubini, Algorithms for Communications Systems and their Applications (Wiley, 2002). 


\section{University Library}

\section{- M M N E R VA A gateway to Melbourne's research publications}

Minerva Access is the Institutional Repository of The University of Melbourne

Author/s:

Li, J;Lim, C;Nirmalathas, A

Title:

Indoor Optical Wireless Communications using Few-mode Based Uniform Beam Shaping and LMS Based Adaptive Equalization

Date:

2020-09-01

\section{Citation:}

Li, J., Lim, C. \& Nirmalathas, A. (2020). Indoor Optical Wireless Communications using Few-mode Based Uniform Beam Shaping and LMS Based Adaptive Equalization.

Proceedings of IEEE Photonics Conference, IPC 2020, 00, IEEE. https://doi.org/10.1109/ IPC47351.2020.9252273.

Persistent Link:

http://hdl.handle.net/11343/258811 